\title{
El problema del texto en la investigación evaluativa: Un estudio de caso
}

The problem of text in evaluative research: A case study

\author{
Ángel Rivero Recuenco \\ Universidad de Alcalá (Madrid) \\ angel.rivero@uah.es (ESPAÑA)
}

Recibido: 08.112016

Aceptado: 11.07.2017

\section{RESUMEN}

Sobre la base de una retórica de la evidencia, los discursos tecnocráticos construyen tramas narrativas "protagonizadas" por medidas e índices incuestionables, ante los que no caben argumentaciones. De este modo, se presentan determinadas decisiones como las únicas posibles, como el one best way de la acción de gobierno. Ante el texto de un informe que se presenta en términos de verdad científica y política, se propone un análisis de sus procesos de elaboración, de sus formas de apropiación y de sus usos; una crítica de sus condiciones de posibilidad que tome como objeto el propio texto del informe. Este artículo proporciona una muestra de ese trabajo crítico. Mediante el estudio intensivo de la evaluación de una intervención pública, se analiza el proceso de producción de un texto evaluativo, su resultado discursivo y su recepción institucional. De ese análisis se derivan algunas implicaciones acerca de la investigación como proceso de escritura y de los juegos de acoplamiento y desacoplamiento entre discursos-prácticas de investigación y discursos-prácticas gubernamentales.

\section{PALABRAS CLAVE}

Evaluación de políticas. Gubernamentalidad. Estudios críticos de gestión. Análisis del discurso. 


\begin{abstract}
Based on a rhetoric of the evidence, technocratic discourses construct narrative plots «starring» by unquestioned measures and indicators before which counter arguments do not fit. Thus, certain decisions are presented as the only possible ones, as the one best way of government action. Before the text of a report presented in terms of scientific and political truth, we propose an analysis of its elaboration processes, its forms of appropiation and its uses; a critique of its conditions of possibility whose object is the text of the report itself. This article provides a sample of this critical work. Through intensive study of the evaluation of a public intervention, we analyze the production process of an evaluation report, its discursive result and its institutional reception. From that analysis, we derive some implications about research as writing process and about the coupling and decoupling games between research and government discourses and practices.
\end{abstract}

\title{
KEY WORDS
}

Evaluation of policies. Governmentality. Critical management studies. Discourse analysis.

\section{INTRODUCCIÓN}

La constitución de la evaluación como un saber específico, distinguible de otras formas de investigación social empírica, acontece en los Estados Unidos. Su historia arranca de la psicometría de fines del siglo XIX para dar lugar, hacia mediados del siglo XX, a una práctica centrada en la medición del logro de los resultados esperados por los gestores de intervenciones (originalmente de carácter educativo) que requerían ser valoradas. Estos resultados esperados se denominarán objetivos y las intervenciones, programas. El modelo tyleriano de medición de objetivos (Smith y Tyler, 1942; Tyler, 1950) da origen a la evaluación de programas, y por tanto a la evaluación como disciplina autónoma.

Este modelo se va a complejizar en las siguientes décadas, desembocando en el llamado enfoque sistemático (Rivlin, 1971; Rossi, Freeman y Wright, 1979) y en la aplicación, con propósitos evaluativos, de métodos experimentales y cuasiexperimentales de investigación social (Campbell, 1969, 1975; Rivlin y Tympane, 1975). Después, en la década de 1980, la evaluación tenderá a aproximarse a una técnica de gestión por objetivos que se diversificará, a su vez, en distintas modalidades de análisis de eficiencia y coste-resultado (Quade, 1982; Reynolds y Gaspari, 1985). 
El paradigma dominante en evaluación se forma siguiendo este vector histórico, de raíz cientifista y gerencial ${ }^{1}$, que va de la psicometría a los análisis costeresultado. Un paradigma hegemónico que constituye el "campo de oposición" contra el que se van a forjar diversas concepciones alternativas de la práctica de la evaluación que, a su vez, se ramificarán en una extensa red de elecciones teórico-metodológicas: enfoques participativos, dialógicos, basados en el estudio de casos (Stake, 1976, 1978; Stake y Easley, 1978), democrático-liberales y orientados a la justicia social (House, 1980), pospositivistas (Campbell, 1982), constructivistas (Guba y Lincoln, 1986, 1989), democrático-deliberativos (House y Howe, 1999), etc.

Las escisiones que se suceden en el campo de la evaluación son coherentes con las rupturas que acontecen en las ciencias sociales a lo largo de las décadas de 1970 y 1980: inserción fenomenológica, giro interpretativo, maridaje con la hermenéutica... A partir de estas rupturas, se produce una crítica de la evaluación como actividad eminentemente científica, correlativa a una concepción metodológica de esta como apertura, encuentro y diálogo con los sujetos evaluados.

El objetivismo, derivada extrema de la noción científico-positiva de objetividad, constituye el núcleo crítico de las difracciones teórico-metodológicas que se producen en el campo evaluativo a partir de la década de 1970. Para autores como Scriven (1972), House (1980) o Guba y Lincoln (1989), el objetivismo provoca tres desplazamientos perversos:

1. Sustituye la noción de validez por la de fiabilidad. La medición precisa, reproducible, basada en el acuerdo entre observadores, es su horizonte de idealidad.

2. Sustituye la imparcialidad del evaluador por la neutralidad, una especie de derivada ético-política de la objetividad, conceptuada en esos mismos términos de reproducibilidad de las observaciones por acuerdo intersubjetivo.

3. La responsabilidad moral del evaluador respecto a sus juicios y a los usos dados a la evaluación desaparece; se sustituye por un compromiso metodológico, sustentado en un consenso procedimental-formal. Si este consenso técnico y experto se alcanza, se acepta la validez de los resultados de la evaluación (y los cursos de acción derivados de estos). Entonces, como se dice comúnmente, "los datos hablan por sí mismos"2.

${ }^{1}$ Gerencial en un doble sentido: en primer lugar, por su dependencia de conceptos y prácticas procedentes del management y que remiten a las funciones de planificación, dirección y control asignadas a los responsables de gestión de una organización. En segundo lugar, por su unilateralidad: en los enfoques gerencialistas de evaluación no hay participación activa de otros actores que no sean las gerencias que promueven, financian o administran las intervenciones evaluadas. Sobre la crítica del gerencialismo en evaluación, Cfr. Scriven (1973), House (1980) y Guba y Licoln, (1989).

2 Dicho por Guba y Lincoln (1989: 38): "la adhesión al paradigma científico exonera al evaluador de toda responsabilidad moral por sus acciones. Uno no puede ser culpado sólo por decir la verdad (...). Es fácil argumentar que el evaluador no puede controlar cómo los resultados de una evaluación son utilizados. Es también fácil afirmar que el evaluador no tiene responsabilidad en el seguimiento de una evaluación; su rol termina cuando el informe es entregado. En ninguna 
La crítica del objetivismo implica una crítica política en la que está en juego una determinada concepción del poder y la democracia. A ello subyace un imperativo pragmático: la acción que la evaluación está llamada a movilizar. Esta debe estar colectivamente determinada, no delegada en un gestor técnico (crítica de la tecnocracia), ni en un cargo electo (crítica de la democracia representativa, preferencial, apelación a una democracia participativa, deliberativa). El objetivismo es la coartada de la unilateralidad en las decisiones político-gerenciales, a las que legitima por el lado de la ciencia. Una fractura fundamental va a atravesar el dominio de la evaluación: la que contrapone los enfoques de tipo científico-gerencial con los de carácter pluralista, participativo, dialógico (enfoques de difusión del poder, en la terminología de House; paradigma de la negociación, según Guba y Lincoln) ${ }^{3}$.

El texto de los informes evaluativos se constituye, entonces, en problema. Frente al informe técnico convencional, concebido como un mensaje que refleja lo que existe en la naturaleza, se proponen modos alternativos de concebir los textos, nuevos modos de validarlos y de usarlos:

- Un informe es, necesariamente, un artificio, una construcción socialmente condicionada. La realidad "tal como es" no puede reflejarse en ningún informe ${ }^{4}$. En el límite, la realidad misma es construcción social: se produce mediante procesos dialógicos, y el texto del informe es el relato de esos procesos (Guba y Lincoln, 1989) ${ }^{5}$.

- Un informe debe ser la narración idiosincrásica de un caso en el que sus actores se reconozcan (Stake, 1976, 1978). Se busca un consenso sustantivo que involucre a estos, no un consenso formal-procedimental entre especialistas externos.

- La objetividad del informe no se halla en la reproducibilidad de las observaciones realizadas. Scriven $(1972,1976)$ propone una objetividad cualitativa, basada en el control de sesgos.

- La validez se disocia de la fiabilidad. House (1980) la sitúa en el texto mismo (consistencia interna de la narración, coherencia entre recursos estilísticos, descripción cualitativa y análisis cuantitativo...), y en su recepción (congruencia del texto con el marco experiencial de los sujetos evaluados). El informe debe ser comprensible y creíble para sus

\footnotetext{
circunstancia el evaluador (mensajero) puede ser tenido por responsable de unos resultados (el mensaje) que simplemente reflejan lo que existe en la naturaleza."

${ }^{3}$ Las raíces de los enfoques participativos y dialógicos en evaluación están en los trabajos de Robert Stake (1976, 1978). El enfoque constructivista-respondiente de Guba y Lincoln (1989) llevará hasta sus últimas consecuencias la serie teórica abierta por Stake.

${ }^{4}$ En palabras de House (2000 [1980]: 97): "la realidad tal como es (...) siempre será algo indeterminado. El evaluador se enfrenta con un universo ambiguo. Gracias a diversas lentes sociales y psicológicas, el evaluador representa esos hechos en un informe. El informe en sí es un producto artificial".

5 Dicho en palabras de Shaw (2003 [1999]: 66): “Un informe es simplemente el 'residuo' que dejó el proceso que creó los 'hechos"'.
} 
destinatarios.

- El informe deja de ser un texto que dice lo que necesariamente debe hacerse en virtud del rigor de un método y de la verdad que este proporciona. El texto es un instrumento, un referente dialógico. Se debe usar para dialogar con él y sobre él. Para House y Howe (1999) este diálogo es la base de una deliberación racional de la que derivar la acción.

El interés actual de estos debates no opera tanto sobre el plano epistemológico o metodológico como sobre el político. Su relevancia es, ante todo, de orden pragmático. Remite al uso político del conocimiento técnico. Es en el contexto de un uso tecnocrático de los textos donde los supuestos objetivistas continúan operando de modo políticamente coactivo.

El discurso tecnocrático construye tramas narrativas "protagonizadas" por medidas e índices supuestamente inapelables (hechos "puros y duros", ante los que no caben argumentaciones). Es así, sobre la base de una "ideología de la evidencia", como se presentan determinadas decisiones como las únicas posibles, como el one best way de la acción de gobierno. En el ámbito de las ciencias sociales (particularmente de la economía, aunque no solo) es donde esa retórica de lo dado se exhibe de modo especialmente activo.

Ante el texto de un informe que se presenta en términos de verdad científica (y política), se impone un análisis de sus procesos de elaboración, de sus formas de apropiación, de sus usos... Una crítica de sus condiciones de posibilidad que tome como objeto, como caso, el propio texto del informe.

Este artículo proporciona una muestra de ese trabajo crítico. Sobre la base de un caso, mostramos el proceso de producción de un texto evaluativo, su resultado discursivo y su recepción institucional. De la descripción densa del caso derivamos algunas implicaciones acerca del lugar del texto en las prácticas de la investigación evaluativa (y, más en general, de toda investigación que afecte a cuestiones de interés público).

Tal vez sea un lugar común que un informe técnico es una construcción, una escritura políticamente condicionada, no un reflejo especular del mundo. Sin embargo, así es presentado y usado políticamente. La descripción del caso permite observar cómo acontecen esos procesos de construcción y apropiación, y qué sucede cuando la ortodoxia de las prácticas de evaluación/investigación se alteran.

${ }^{6}$ La expresión es del antropólogo francés Marc Augè (2004: 176). 


\section{ANÁLISIS DEL CASO}

\subsection{ENFOQUE DE INVESTIGACIÓN}

El caso se inscribe en una investigación de mayor alcance, cuyo propósito general fue realizar una crítica política del saber evaluativo 7 . Dicho brevemente, quisimos determinar en qué condiciones, a partir de qué supuestos y con qué efectos se producen los juicios establecidos por medio de la evaluación ${ }^{8}$.

En el plano teórico, el enfoque de la investigación incorpora una doble mirada, formada por las categorías y orientaciones de método propias de la crítica foucaultiana (Foucault, 1969, 1981, 2004), por un lado, y la teoría de la hermenéutica de Paul Ricoeur $(1969,1971,1986)$, por otro. Desde esta doble perspectiva, indagamos en cuatro aspectos o problemas concernientes a la práctica evaluativa:

1. El problema de sus condiciones (discursivas, epistemológicas, políticas) de posibilidad.

2. La cuestión de los efectos de esta práctica.

3. Su integración en un campo de relaciones de poder.

4. El problema de los procesos de apropiación/recepción de los textos evaluativos, de donde se derivan los usos finales y la eficacia política de la evaluación.

En el plano metodológico, los principales rasgos de la investigación han sido los siguientes:

- Eminentemente cualitativa, en su orientación general.

- Centrada en el análisis de textos (informes de evaluación, documentos administrativos, metodológicos y de planificación) y discursos (entrevistas abiertas con informantes cualificados).

- Basada en el estudio intensivo de casos como estrategia vertebradora de la investigación, especialmente apropiada para obtener un conocimiento empírico detallado de la práctica evaluativa.

El caso central de la investigación es el de la evaluación del Fondo de Apoyo para la Acogida y la Integración de los Inmigrantes (FAIRE), llevada a cabo entre los años 2007 y 2008 a instancia de la Dirección General de Integración de los Inmigrantes de la Secretaría de Estado de Inmigración y Emigración del Ministerio de Trabajo y Asuntos Sociales. El FAIRE constituyó, hasta su sus-

${ }^{7}$ Tomamos de Michel Foucault el concepto de "crítica política del saber" (Foucault, 2009 [2004]: 46-47).

${ }^{8}$ La investigación se desarrolló como tesis doctoral entre los años 2012 y 2015. La tesis (Más allá de la eficacia. Crítica y hermenéutica de la evaluación) fue defendida en junio de 2015 en la Facultad de Ciencias Políticas y Sociología de la UNED. 
pensión en 2012, el principal instrumento de financiación para el desarrollo de la política de integración de inmigrantes. Su evaluación estuvo a cargo de una empresa especializada en este tipo de trabajos, seleccionada mediante el preceptivo concurso público.

Varias razones justifican la selección de la evaluación del FAIRE como caso central de la investigación:

- Su significación para el estudio de la institucionalización de la evaluación en el ciclo de una política estatal. La evaluación del FAIRE no estuvo instada de manera externa por un organismo financiador supranacional (como solía ser habitual), sino que fue promovida de forma autónoma por el Estado, en el contexto de un proceso más amplio de reforma gerencialista de las Administraciones públicas, puesto en marcha por el primer Gobierno del Presidente José Luis Rodríguez Zapatero9.

- Fue una evaluación que involucró a diferentes actores y niveles de gobierno, y en ella se ensayó una metodología de inspiración constructivista-respondiente, cuya aplicabilidad e implicaciones sociopolíticas poseen un notable interés ${ }^{10}$.

La particularidad de la evaluación del FAIRE se deriva, sobre todo, del juego político en que se inscribió y de las elecciones teórico-metodológicas que se hicieron. El inusual intento de aplicación del enfoque constructivista-respondiente para evaluar una intervención estatal de amplio alcance hace del caso un analizador especialmente interesante de la práctica evaluativa, de sus condiciones y de sus límites.

Podríamos decir que es en la extrañeza de esta evaluación, en su "anomalía metodológica", donde se revela, como por contraste, lo normal. Las normas de la evaluación en tanto que práctica discursiva fijada por la escritura.

\subsection{RESUMEN DE RESULTADOS DE LA INVESTIGACIÓN}

La estructura analítica del caso se compone de tres elementos: contexto, texto y recepción. El centro del análisis es el texto del informe de evaluación, pero partimos de unos contextos, nunca ausentes del texto y que se revelan energéticamente al final, en la recepción del informe.

En el análisis de los contextos, distinguimos entre un contexto sociopolítico global y un contexto local, relativo a la formación de la demanda del informe y su proceso de producción ${ }^{11}$.

9 Sobre este proceso de reformas y el lugar de la evaluación en el mismo, Cfr. Garde (2006).

${ }^{10}$ Según Shaw (2003 [1999]: 67): "Aunque no hay duda de que el paradigma de Guba y Lincoln ha cambiado nuestras ideas sobre la evaluación, necesitamos ejemplos de su aplicación para desarrollar y juzgar su validez".

${ }^{11}$ La distinción entre contexto global y contexto local está tomada de Van Dijk (2000). 


\subsubsection{Contexto global: la política de integración de inmigrantes}

Desde su origen, varios ejes de tensión atraviesan el ámbito político de la integración de inmigrantes ${ }^{12}$. Destacamos dos:

- La política se constituye frente a unas prácticas de gobierno basadas en el control de los flujos migratorios. A la presión migratoria se ha respondido históricamente, ante todo, con el control de la frontera, la represión del flujo. La integración de los inmigrantes debe encontrar su espacio en el marco de una política de seguridad interior regulada por una Ley que determina las posibilidades de acogida de los extranjeros (y, por tanto, también su exclusión). Para muchos de quienes participaron en los debates previos a la formación de la política (profesores, juristas, representantes de organizaciones sociales...), la Ley de extranjería va a ser puesta en cuestión. Va a haber una tensión constante entre acogidaintegración social y control de fronteras-represión de flujos.

- La distribución territorial del poder en el Estado autonómico hace residir las competencias en materia de integración de inmigrantes en las regiones y, secundariamente, en los municipios. Sin embargo, la política se financia principalmente desde la Administración central, que va tratar de controlar y dotar coherencia programática a las actuaciones llevadas a cabo por los agentes territoriales. Una tensión competencial atravesará la implementación de la política de integración de inmigrantes.

La evaluación del Fondo de Apoyo, y la escritura del texto del informe, se producen en este marco contextual, en este campo de discursos y prácticas que, excediendo al caso, lo contienen. La problemática del contexto global va a determinar el proceso de la evaluación y sus resultados.

\subsubsection{Contexto local: El proceso de la evaluación}

Lo que hemos llamado contexto global constituye el marco de inscripción del contexto local del caso, aquel en el que se produce la decisión de evaluar el FAIRE, se determina su demanda y se lleva a cabo la escritura del informe.

En la formación de la demanda de evaluación distinguimos:

${ }^{12}$ Una muestra significativa de los debates que acompañaron la formación de la política estatal de integración de inmigrantes, y la propia creación del FAIRE, fueron los seminarios Inmigración y sociedad y Justicia, tolerancia e inmigración, auspiciados por la Fundación Pablo Iglesias y desarrollados entre 2004 y 2005 en Madrid. En su conjunto, constituyen una excelente muestra de las cuestiones suscitadas y de las inquietudes sentidas ante el fenómeno migratorio, en el entorno del Gobierno que estaba diseñando la política en cuestión, por especialistas académicos, cargos políticos y representantes de las organizaciones sociales. Las ponencias presentadas en estos seminarios, así como las transcripciones de los debates que suscitaron, se recogen en la edición coordinada por Rafael del Águila (2005). 
- El requerimiento del cliente, la Dirección General de Integración de los Inmigrantes (DGII).

- La demanda latente de la DGII.

- La constitución técnica de la demanda: el diseño de la evaluación elaborado por el evaluador ${ }^{13}$.

En la formación de la demanda, y en el proceso material de realización de la evaluación, tiene lugar un juego de acoplamientos evaluación-dispositivo gubernamental. Bajo la capa de unos consensos más aparentes que reales, la transformación del requerimiento en diseño técnico encubre acoplamientos fallidos y desfases. En un principio, esos desfases resultan casi imperceptibles, pero se van a ir haciendo progresivamente visibles a lo largo del proceso evaluativo. El caso muestra la reproducción encadenada de una serie de tensiones que se trasladan del contexto global al contexto local de la evaluación, a su proceso de realización y a la escritura del informe. Su manifestación más patente, más energética, se producirá en el momento de la recepción institucional del texto.

El requerimiento del cliente era, en origen, muy genérico; hubiera podido valer para cualquier evaluación de cualquier política ${ }^{14}$. Pero, bajo el esquematismo del requerimiento, latía una demanda de legitimación institucional y de modulación de las relaciones de poder que atravesaban el campo de la política de integración ${ }^{15}$. Había un desajuste material de base, ya comentado: la DGII diseña la política y la financia, pero son los agentes territoriales quienes poseen las competencias y ejecutan la política. La tensión competencial, decíamos, condiciona el proceso de la evaluación.

El evaluador interpretó el requerimiento y la demanda latente de la DGII y los convirtió en demanda técnica, en diseño de evaluación ${ }^{16}$. Trabajó en las distintas dimensiones del requerimiento: ¿quién iba a utilizar la evaluación, para qué y cómo?

${ }^{13}$ La distinción entre requerimiento y demanda está tomada de Ibáñez (1986).

${ }^{14}$ Según el Pliego de condiciones técnicas anexo a la publicación del concurso público que regulaba la contratación de la evaluación (BOE de 24 de enero de 2007), los objetivos de esta fueron: "Valorar la pertinencia, validez y eficacia de las medidas y actuaciones (...). Evaluar el efecto del Fondo de Apoyo en el proceso de integración de los inmigrantes (...). Obtener conclusiones y recomendaciones para la eficaz continuidad del Fondo."

15 En las entrevistas realizadas emerge reiteradamente un propósito de visibilizarse como experiencia ejemplar: "La Unión Europea nos había seleccionado como un proyecto de buenas prácticas. Entonces, al ser un proyecto de buenas prácticas había que introducir elementos de evaluación" (Entrevista cargo directivo de la DGII). Ya en la Exposición de motivos del Marco de cooperación para la gestión del Fondo de Apoyo, 2006 (un documento en el que se fijaban los objetivos y principios de la intervención para ese año), se incluyó una referencia a esta selección ejemplar del FAIRE por parte de las instituciones comunitarias, previa a cualquier evaluación.

${ }^{16}$ El diseño se hizo explícito en el documento denominado Diseño del modelo de evaluación (GPI, 2007), presentado a las Comunidades Autónomas el 27 de junio de 2007. No obstante, como veremos, este diseño original se fue modificando a lo largo del proceso evaluativo. 
Respecto al quién, para el evaluador, todos los agentes involucrados en la política debían ser partícipes y usuarios de la evaluación. El cliente estuvo de acuerdo. De hecho, quería que la evaluación fuera participativa, pero solo hasta cierto punto y bajo ciertas condiciones. Los usos finalmente dados al texto del informe mostrarán a los "verdaderos" propietarios del texto y de la función evaluativa, quiénes tendrían derecho a leer el informe y a usarlo.

Respecto al para qué, el evaluador reconsideró los fines del Fondo de Apoyo y propuso una evaluación orientada fundamentalmente a valorar la organización creada para implementar la intervención, sus procesos de gestión, las pautas de relación interadministrativa desarrolladas y los efectos políticos e institucionales derivados de todo ello (los efectos sobre la integración de los inmigrantes quedaron relegados a un segundo plano). Hay aquí un acoplamiento parcial con la demanda del cliente. La DGII manifestó su acuerdo en centrar la evaluación en los aspectos organizativos y de gestión, pero lo que esperaba, ante todo, era poner en valor el modelo establecido y legitimarse: frente a los agentes territoriales, la Administración central y la Unión Europea ${ }^{17}$.

Asimismo, el evaluador propuso que la evaluación sirviera para rediseñar la política mediante un proceso de negociación y diálogo con los agentes. Hay aquí un primer desencuentro manifiesto: la DGII consideraba que la participación de los agentes no debía llegar tan lejos. Estos debían estar presentes en el proceso de la evaluación, pero no les correspondía participar en la redefinición del programa de la intervención ${ }^{18}$.

La resolución del cómo hacer la evaluación desembocó en la adopción de un doble soporte teórico-metodológico:

1. Por un lado, el evaluador propuso realizar el análisis de la organización y los procesos a partir de la teoría de la agencia (Jensen y Mecklin, 1976), un enfoque eminentemente gerencial, centrado en el análisis de la relación gerencia-agentes ${ }^{19}$.

${ }^{17}$ En las entrevistas, los responsables del Fondo recuerdan la complacencia sentida respecto a lo que habían hecho, la convicción de que la evaluación iba necesariamente a confirmar su imagen positiva y ejemplar del modelo creado: "Había que hacer una evaluación a partir del modelo que habíamos creado (...). Por lo tanto, en el modelo había que tener presentes los tres niveles de las Administraciones para valorar qué había significado el Fondo para cada una de ellas (...). [E]stábamos convencidos de que el resultado siempre iba a resultar positivo" (Entrevista cargo directivo de la DGII).

${ }^{18}$ En un documento, denominado Observaciones de la DGII a documento Diseño metodológico de la evaluación, Borrador I, de fecha 28 de mayo de 2007, dirigido a los evaluadores, los responsables de la DGII expresaron sus reservas ante las implicaciones de una metodología participativa: "Aclarar sobre la participación de los actores clave (...). Delimitar 'su implicación activa en la redefinición de la nueva programación del Fondo de Apoyo'. Lo que se desea plantear principalmente es su participación en la evaluación desde el inicio y no en la redefinición de la nueva programación."

${ }^{19}$ La teoría de la agencia se consideró "útil para describir y analizar las situaciones en las que el responsable político de una actividad (el principal) encarga a uno o varios agentes desarrollarla, siendo funciones del principal establecer los objetivos de la actividad a desarrollar por el agente, fijarle los incentivos (...) y controlar su desempeño” (GPI, 2007: 9). 
2. Por otro lado, en el plano metodológico, el evaluador se apartó de las normas predominantes en el campo de la evaluación. Propuso un diseño participativo y dialógico basado en el enfoque constructivista-respondiente de Guba y Lincoln (1989), que se articularía mediante la realización de reuniones de grupo y entrevistas con los agentes territoriales. La DGII quería, en efecto, que los agentes participasen. Pero el evaluador interpretó el requerimiento de participación del cliente en términos de máximos, asumiendo una concepción hiperigualitaria de las relaciones entre los diferentes grupos afectados por la evaluación ${ }^{20}$. El evaluador sobre-interpretó el requerimiento.

Lo que la DGII quería era una participación limitada, controlada, que, mediante la regresión a una grupalidad primaria, "acogedora", favoreciera la cohesión del campo gubernamental, la atenuación de sus energías centrífugas, no su estímulo $^{21}$. La participación no se quería para definir conjuntamente una realidad ni para modificar el modelo de intervención del FAIRE. Se quería a los agentes como suministradores de información y testigos de un proceso que se deseaba validar.

Aparentemente, había articulación entre el diseño técnico del evaluador y la tecnología de gobierno que regía la intervención evaluada:

- La teoría de la agencia correlaciona con una tecnología de gobierno descentralizada que necesita asegurar el control de la relación donantereceptor de recursos. Hay acoplamiento ideológico entre la teoría y los postulados de la Nueva Gestión Pública, base de la reforma gerencial de las Administraciones públicas que se estaba promoviendo a una escala más amplia en el conjunto del Estado 22 .

- El enfoque constructivista-respondiente correlaciona formalmente con esa misma tecnología en el plano de la participación. En el marco de una gubernamentalidad descentralizada, se requiere la cooperación de los agentes, una participación activa en la ejecución de la política, cierto grado de corresponsabilidad financiera (todo ello en los límites de la adhesión a los objetivos y principios fijados desde la gerencia central).

Son ajustes aparentes, acoplamientos teóricos y formales. En el proceso de la evaluación se harán evidentes los desencuentros. En las primeras reuniones de

${ }^{20}$ Sobre las derivadas del paradigma constructivista en evaluación (el hiperigualitarismo entre otras), Cfr. House y Howe (1999).

${ }^{21}$ Para la DGII era muy importante que los agentes se sintieran "cómodos" en el proceso participativo. Así lo advirtió enseguida el evaluador: "[P]reocupan a la DGII algunos aspectos relativos al ambiente y al clima relacional (...) de las reuniones. Para la DGII las reuniones (...) constituyen una oportunidad para el encuentro, el intercambio (informal) de información, el fortalecimiento de las relaciones, etc. (...)" (Informe de situación 29.05.2007: 2).

${ }^{22}$ Sobre las reformas gerenciales de las Administraciones y el ideario (neoliberal) de la llamada Nueva Gestión Pública, Cfr. Du Gay $(1994,2000)$. 
grupo con representantes de las Comunidades Autónomas se pone de manifiesto el conflicto. La realización de los grupos desembocó en un cuestionamiento de las atribuciones competenciales de la DGII y de la propia pertinencia de la evaluación ${ }^{23}$.

El resultado de este conflicto fue la alteración del diseño participativo originalmente propuesto: se suspendieron las reuniones con los agentes y, finalmente, se restringirá la difusión del informe al entorno gerencial de la DGII.

El informe de evaluación del Fondo de Apoyo (GPI, 2008a) va a fijar, en la materialidad de su escritura, el juego de acoplamientos y desacoplamientos que se dieron en los procesos de formación de la demanda y realización de los trabajos de campo de la evaluación.

\subsubsection{El texto}

El análisis del texto del informe se articula en tres ejes, relativos a:

1. El régimen discursivo a partir del cual se producen los enunciados de la evaluación.

2. Los efectos textuales de creación de valor y de devaluación, de legitimación y deslegitimación.

3. La lógica estratégica que subyace al texto y los sentidos que de esa lógica se derivan.

\section{Régimen discursivo}

En el texto, se observa la huella de una serie de operaciones que determinan el régimen que hace posible la formación de los enunciados evaluativos ${ }^{24}$. Esas operaciones se refieren a:

- La formación de los objetos de los que "habla" el texto.

- La formación de los conceptos y criterios a partir de los cuales se describen y califican esos objetos.

- La adopción de las elecciones teóricas y metodológicas que configuran el régimen epistemológico del informe.

${ }^{23}$ En la primera de las reuniones de grupo, celebrada el 11 de julio de 2007 con representantes de las dependencias autonómicas competentes en materia de integración de inmigrantes, un sector de los asistentes manifestó su oposición a la celebración de la reunión y a la realización de la evaluación: "[N]o estamos en capacidad de permitir que un organismo distinto a la Comunidad (...) dicte cuáles son las prioridades en la integración de los inmigrantes (...) y creo que somos varias las Comunidades Autónomas que entendemos que no puede legítimamente indicarse si nuestro proceder de cara a la integración de los inmigrantes es oportuno o inoportuno, si es pertinente o no" (Reunión de grupo con representantes de Comunidades Autónomas).

${ }^{24}$ Seguimos a Foucault (1969). 
El régimen enunciativo del texto no es una mera reproducción de lo escrito en el diseño metodológico previo. Este se ha ido reconfigurando a lo largo del proceso de la evaluación y en la propia escritura del informe, donde determinadas decisiones se justifican y sobre-justifican. Se observan en el texto nuevos conceptos, una mayor precisión en el análisis de los aspectos institucionales y económicos del objeto evaluado. La problemática de la evaluación del FAIRE se conceptúa, casi exclusivamente, como problemática organizativa y financiera, problemática de gestión.

Los objetos del texto se constituyen, como es lógico, sobre la superficie de inscripción de la política de integración de inmigrantes y su principal instrumento: el Fondo de Apoyo. Sobre este referencial genérico, los objetos se delimitan desde las instancias tecno-científicas de la economía de la empresa, el análisis de las organizaciones y el management. Una serie de categorías y matrices de clasificación distinguen y especifican los objetos: actividades programadas, recursos y objetivos; procesos estratégicos, de soporte y operativos; efectos de integración institucional, de creación de estructuras, de refuerzo y complementariedad financiera... La hipóstasis de lo formal-instrumental es el rasgo más llamativo de la construcción del mundo-objeto del texto. Lo organizativo y lo tecno-económico delimitan el dominio de validez de la evaluación ${ }^{25}$.

Lo sustancial de la intervención evaluada, las necesidades de acogida de los inmigrantes, las problemáticas sociales de la integración, han quedado definitivamente marginadas del decir del texto, desplazadas por la lógica formalinstrumental que rige su escritura. La acogida y la integración social constituyen una especie de referencial secundario (casi un pre-texto) sobre el que se proyectan los temas que se tratan en el informe. Es la re-integración de las agencias de gobierno, la vertebración de un dispositivo gubernamental, su funcionalidad y su eficacia institucional lo que se constituye en objeto principal de discurso.

Los conceptos manejados se delimitan desde las mismas instancias tecnocientíficas de la economía y el management. Su aplicación sobre los objetos del texto produce los juicios evaluativos. El ajuste es la operación preconceptual que da forma a los enunciados de la evaluación. Los conceptos que articulan la secuencia expositiva del informe (coherencia formal, funcionalidad de los procesos, eficacia, pertinencia) permiten establecer relaciones de ajuste entre los objetos. La verdad de la evaluación es la verdad lógica y empírica de esas relaciones de ajuste: entre objetivos, actividades y recursos programados (juicios de coherencia formal), entre procesos y resultados (juicios de funcionalidad), entre

${ }^{25}$ El informe se organiza en siete capítulos: I. Enfoque de la evaluación, II. El Marco de cooperación: diseño y evaluabilidad, III. La intervención: organización y procesos, IV. Eficacia, V. Pertinencia, VI. Conclusiones, y VII. Recomendaciones. Las referencias de extensión son indicativas: el capítulo más amplio, con diferencia, es el dedicado a organización y procesos (ocupa treinta y seis páginas, prácticamente un tercio del informe, superando notablemente a los demás capítulos, cuya extensión dobla o incluso llega a cuadruplicar). En los otros capítulos es también muy notable la presencia de temáticas de carácter financiero y organizativo. 
efectos logrados y objetivos (juicios de eficacia), entre objetivos y necesidades (juicios de pertinencia). El Fondo de Apoyo se concibe como una máquina cuyo ajuste ha de ser evaluado, calificado en la escritura.

Hay continuidad entre lo conceptual y lo axiológico: los conceptos manejados son, asimismo, los criterios de valor de la evaluación. Su aplicación sobre los objetos se representa mediante series de significantes que pertenecen, dadas las instancias de delimitación manejadas, al campo semántico del management. Dos categorías vertebran la argumentación evaluativa: control de eficacia y sistema de incentivos. En el texto se valora si el Fondo fue un incentivo de desempeño y si, en tanto tal, estimuló la eficacia y la eficiencia de los agentes para lograr mejores resultados y obtener, como recompensa, más financiación del Ministerio. Una de las principales ideas-fuerza del informe, constantemente reiterada, es que la financiación de los agentes debía estar ligada al logro de resultados ${ }^{26}$.

La estructura axiológica fundamental del texto remite, por tanto, al gerencialismo. Los valores afirmados (eficacia, eficiencia, excelencia en la gestión...) articulan con el proceso de reforma que, en el momento de realización de la evaluación, se está implantando en las Administraciones públicas.

No obstante, el régimen teórico-metodológico desde el que se construye el texto es dual. Delimita una encrucijada en el dominio discursivo de la evaluación, un punto en el que se enfrentan y difractan dos concepciones de la práctica evaluativa: el gerencialismo, sostenido por la elección de la teoría de la agencia como perspectiva del análisis de la organización y los procesos, y el pluralismo, fundado en la elección del enfoque constructivista-respondiente como forma de articular la participación. Esta última elección induce una apertura descentradora de la unilateralidad del punto de vista gerencial. La apertura del texto al discurso de los agentes territoriales lleva a la escritura del informe otras constelaciones de valor: valores relativos a la integración social de los inmigrantes, que desplazan la centralidad de los criterios eminentemente instrumentales y gerenciales relativos a la funcionalidad de los procesos y la eficacia institucional del Fondo ${ }^{27}$.

Como decíamos antes, hay articulación aparente entre el régimen epistemo-

${ }^{26}$ En el extenso capítulo III del informe (Organización y procesos), y en las conclusiones finales, se valora si, en la gestión del FAIRE, hubo "un verdadero control de eficacia que sirviera para hacer una correcta asignación de incentivos en función de los logros"; un sistema de control y de incentivos que sirviera para "redefinir metas (misión), reprogramar (reasignación de recursos) y reorientar los procesos con el fin de mejorar la eficacia, eficiencia y calidad de la intervención" (GPI, 2008a: 44-45). La respuesta a esta cuestión constituye uno de los ejes fundamentales en la argumentación evaluativa del informe.

27 Aunque este desplazamiento axiológico es limitado. Acontece al final, en el capítulo V (Pertinencia), y de forma indirecta: por medio de la transcripción de "las discrepancias acerca del modelo apreciadas por los actores implicados en la intervención" (GPI, 2008a: 7). En el capítulo I del informe (Enfoque de evaluación), se justifica esta particularidad del material empírico utilizado para valorar la pertinencia. Es una justificación retrospectiva, no aparece en el diseño evaluativo original. 
lógico y metodológico dual del texto y la práctica de una gubernamentalidad basada en la descentralización (controlada) y la participación (limitada). Pero es una articulación engañosa, encubre un acoplamiento fallido, una especie de sobre-acoplamiento. El discurso evaluativo va a llevar más allá de los intereses del cliente los criterios del análisis gerencial y la participación de los agentes.

El resultado de este desacoplamiento entre el discurso evaluativo y los intereses del dispositivo gubernamental que ha instado la evaluación no se observa tanto en el régimen enunciativo del texto como en su actualización, en la materialización de su escritura: en los efectos de valor producidos por el texto y en su polifonía discursiva.

\section{Los efectos de valor}

Encontramos cuatro clases generales de efectos valorativos producidos por el texto: de legitimación política, de devaluación técnica, de cuestionamiento del modelo creado para implementar el Fondo de Apoyo y de cuestionamiento de la Ley de extranjería como instrumento de integración.

En la secuencia expositiva del informe, estas clases de efectos describen un encadenamiento de oscilaciones valorativas, un régimen de alternancia entre la sobrevaloración y la infravaloración de la labor realizada por la DGII: el texto comienza con la legitimación histórico-política de esta dependencia (apartado II.1 del capítulo II: Creación y puesta en marcha del Fondo de Apoyo), a la que suceden su devaluación técnica (a lo largo de todo el capítulo III: Organización y procesos), su legitimación como instancia inductora de políticas regionales y locales (capítulo IV: Eficacia) y, finalmente, el cuestionamiento del modelo de intervención aplicado y de la Ley (en el capítulo V: Pertinencia). Estas oscilaciones valorativas introducen ambivalencia en el texto, cuyo sentido deviene ambiguo, en ocasiones contradictorio. La ambivalencia amenaza con anular la producción de valor que el texto evaluativo está llamado a realizar ${ }^{28}$.

Sin embargo, más allá de las oscilaciones valorativas y la relativa incoherencia del informe, su sentido principal, el querer decir del texto, es la devaluación y la deslegitimación. Los efectos de legitimación que se dan en los capítulos II y IV del informe resultan un tanto forzados. Se contradicen con lo dicho, de forma lógicamente más consistente y empíricamente mejor fundamentada, en los capítulos III y V. En el informe se observa un esfuerzo discursivo muy acentuado por aplanar las contradicciones y mantener la cohesión de su escritura ${ }^{29}$. Pero,

${ }^{28}$ Estas oscilaciones se producen incluso en el seno de un mismo capítulo. Así, en el capítulo IV (Eficacia), la positiva legitimación de los responsables de la DGII, derivada de la afirmada efectividad política del Fondo, es contrarrestada por la enunciación de apreciaciones menos favorables, procedentes de los agentes territoriales (GPI, 2008a: 72-73).

${ }^{29}$ En el citado capítulo IV, donde se describen los logros político-institucionales derivados de la implementación del FAIRE, el evaluador se esfuerza notablemente en relativizar e incluso corregir las apreciaciones críticas de los agentes (GPI, 2008a: 74-75). En general, es muy frecuente en el informe el uso de fórmulas adversativas: "a pesar de..." " más allá de...", "otra cosa es que...", mediante las que el evaluador trata de superar las disonancias del texto. 
más allá de estos esfuerzos, se impone en el texto el relato sobre la incapacidad de la DGII para gobernar con eficacia la política de integración de inmigrantes. En el capítulo V (Pertinencia), el cuestionamiento del modelo diseñado para desarrollar la intervención y, sobre todo, de la Ley de extranjería equivalen a una drástica deslegitimación de la labor realizada. Y, finalmente, en el capítulo VI (Conclusiones), los efectos apologéticos y de legitimación de la DGII se relativizan y atenúan. Se atribuye a dichos logros una provisionalidad derivada de las debilidades organizativas descritas previamente, condicionando la eficacia del Fondo a su corrección.

Los efectos de legitimación de la DGII se explican por la posición del evaluador-autor como sujeto mercantil que tiene que responder al discurso de la demanda y efectuar ciertas concesiones al cliente. Tiene que mantener el equilibrio de la cozy relationship en la que se inscribe la práctica evaluativa ${ }^{30}$. Sin embargo, son concesiones que no contienen ni contrarrestan del todo el decir fuerte, devaluativo y deslegitimador, del texto, cuya fuente es doble:

- Por un lado, radica en la axiología dominante en el informe: hay un "estiramiento" de la lógica gerencial. En cumplimiento de su estatus-rol de sujeto experto, de agente de la racionalidad gerencial, el evaluador lleva al límite esta lógica, dando lugar a la devaluación tecno-instrumental de la DGII ${ }^{31}$.

- Por otro, la apertura intersubjetiva: el enfoque constructivista-respondiente da lugar a una polifonía discursiva que "perfora" la axiología de la gerencia, haciendo que penetren otros valores e intereses. Hay correlación entre apertura metodológica y deslegitimación: la consecuencia última de la apertura intersubjetiva es la deslegitimación política. Su punto álgido se da en la valoración de la pertinencia de la intervención y de la Ley en la que esta se inscribe, momento en que los agentes territoriales se constituyen en la voz dominante en el texto ${ }^{32}$.

${ }^{30}$ El concepto de cozy relationship (relación acogedora), formulado originalmente por Scriven, se refiere a la tácita complicidad comercial que habitualmente se da entre el evaluador y su cliente.

${ }^{31}$ En el capítulo III del informe (Organización y procesos), se argumenta detalladamente que no fue la racionalidad gerencial la que gobernó, en la práctica, el hacer de la DGII. La realidad de la gestión del FAIRE fue otra: una gestión técnicamente ineficaz, condicionada por inercias burocráticas, por tensiones administrativas, partidistas y territoriales que constituyeron un entramado de factores de ineficiencia de los procesos de la intervención. El FAIRE fue una mera "caja pagadora", no un "incentivo de desempeño" (GPI, 2008a: 27). Esta argumentación se reafirma definitivamente en las conclusiones del informe (Ibíd.: 89).

${ }^{32}$ En el apartado V.2.1. (Desajustes identificados) del capítulo V (Pertinencia), los agentes hablan del fenómeno migratorio, las necesidades de la intervención y la adecuación del FAIRE respecto a estas. Su discurso es excepcionalmente crítico: la eficacia del Fondo es irrelevante, pues existe un desajuste radical entre sus objetivos de integración y la Ley de extranjería, que "opera como barrera a la participación en las actuaciones y limita el acceso a los servicios, afectando de manera obvia a las oportunidades de integración social" (GPI, 2008a: 83). Es en este apartado donde se aprecia con mayor intensidad la apertura intersubjetiva de la evaluación. El decir de los agentes autonómicos y locales se sitúa casi siempre en primer plano, llega a hacerse dominante en 
La apertura intersubjetiva de la evaluación lleva a la superficie de inscripción del texto la polifonía del campo político de la integración de los inmigrantes. El contexto se reproduce en el texto. La interpretación final del informe solo es posible mediante el análisis de esa polifonía discursiva y de la lógica estratégica en la que los múltiples sentidos abiertos en la trama del texto se integran.

\section{Los sentidos del texto}

El texto del informe está construido a partir de una pluralidad de posiciones discursivas que trazan múltiples sentidos. Encontramos en el texto discursos centralizadores: el de la gerencia, la DGII, y el del evaluador, como sujeto-agente de la racionalidad gerencial, que se corresponden, en la exterioridad política del texto, con una fuerza centrípeta orientada al control de los recursos del FAIRE y de los agentes territoriales receptores de esos recursos. De forma correlativa, encontramos discursos centrífugos, los de estos mismos agentes, que tratan de escapar de esa fuerza centralizadora, proponiendo legitimidades y centralidades alternativas.

Estos discursos se contraponen y enfrentan entre sí, como el de la DGII y el de los agentes autonómicos y el de estos y los agentes locales. A veces muestran alianzas, zonas de confluencia más o menos estables, aunque limitadas, como la que vincula los discursos de la DGII y del evaluador. Otras veces las alianzas solo se pretenden, como la que buscan los agentes locales con la Administración central en su lucha, prácticamente perdida, contra los agentes autonómicos ${ }^{33}$.

Los discursos que se dan en el texto se despliegan en un juego estratégico en virtud del cual se disputan el uso y el control del FAIRE ${ }^{34}$. En este juego, en su lógica estratégica, los diferentes discursos se integran. En la polifonía discursiva del texto, en el entramado de discursos y contra-discursos que lo constituye, se reproduce el campo de relaciones de poder en el que se inscriben la política de

la escritura del texto. El evaluador les cede, casi por completo, la palabra.

${ }^{33}$ Frente al discurso autonómico, el discurso de la DGII reclama la posición de poder que cree le corresponde, su estatus de instancia central de autoridad, en cuya virtud pretende controlar el uso de lo que considera son sus recursos financieros. El discurso autonómico es su inversión simétrica: cuestiona las pretensiones de control y autoridad que se atribuye la DGII. Frente a los agentes locales, considera que estos no están en condiciones de administrar los recursos del FAIRE y que deben tutelarlos. El discurso local mantiene frente al discurso autonómico una relación asimilable a la que este mantiene con el discurso de la DGII: cuestiona que sean los Gobiernos regionales quienes administren los recursos del Fondo. Frente a la DGII, el discurso local busca una aproximación, una alianza: la DGII debe limitar el desarrollo de esa nueva centralidad regional que se impone sobre los municipios.

${ }^{34}$ Y, por extensión, la distribución territorial del poder. La gestión del FAIRE es un episodio de una lucha competencial más amplia y la evaluación, un motivo táctico más de ella. En el discurso de algunos agentes autonómicos, bajo las reclamaciones competenciales y las críticas proyectadas sobre la DGII, late asimismo una voluntad de desgastar al partido que gobierna (las posiciones críticas más acentuadas proceden del principal partido de la oposición). También está en juego, entonces, el acceso al poder en el conjunto del Estado. Se cruzan una lucha competencial y una lucha de partidos. 
integración de inmigrantes y su principal instrumento financiero, el Fondo de Apoyo.

La heterogeneidad discursiva del texto no se resuelve, por tanto, en la síntesis de una unidad. Pero sí podemos distinguir y jerarquizar tres sentidos fundamentales:

1. En primer lugar, encontramos un sentido manifiesto, aquel que, en el nivel más explícito, parece dominar el texto. Se corresponde con el discurso de la razón gerencial y con el propósito fijado en el diseño de la evaluación: valorar el Fondo de Apoyo desde la clave de la eficacia, prescribir conductas que eleven la funcionalidad de la organización creada. La devaluación técnica que se proyecta sobre la DGII se corresponde con este sentido.

2. Hay un sentido secundario: la legitimación de la gerencia. No domina el decir del texto, pero está presente en él. Responde, como hemos dicho, a la presión del discurso auto-legitimador de la DGII. Se orienta a actualizar el discurso de la demanda, a conceder al cliente una satisfacción que, sin embargo, los otros discursos ahogan.

3. Encontramos, por último, un tercer sentido que, más allá del gerencialismo, la legitimación política de la gerencia y los intereses comerciales del evaluador, atraviesa el texto: la problematización del objeto. El Fondo de Apoyo y la política de integración de inmigrantes se representan en el informe como un referencial saturado de contradicciones, de tensiones administrativas, políticas, sociales... El texto no enmascara los ejes de polarización y las tensiones que atraviesan el ámbito político de la integración de inmigrantes, no mitiga sus contradicciones. Las reproduce discursivamente.

Las razones de esta problematización que, al contravenir tanto los intereses del evaluador como los de la gerencia parece un contra-sentido, hay que buscarlo en la presión de diferentes fuerzas previas al texto. Estas fuerzas actúan sobre el evaluador, que tiene que conjugar un compromiso entre:

- Su sujeción a los principios de la racionalidad gerencial, con los que está vinculado y que debe aplicar para evaluar la gestión del FAIRE (presión del discurso tecnocrático dominante).

- Los deseos que ha de satisfacer para poder seguir existiendo como profesional en el espacio de un mercado (presión del discurso de la demanda).

- Su deber de llevar al texto el discurso de los agentes que él mismo ha convocado (presión de la elección teórico-metodológica).

La problematización del objeto es, ante todo, una derivada de la elección del enfoque constructivista-respondiente como metodología de la evaluación. El evaluador está presionado por esa opción, metodológicamente inusual, y por los discursos que ha desencadenado. Un enfoque estrictamente gerencial hubiera 
circunscrito el sentido del texto a una apreciación técnica de la funcionalidad de la gestión y una cuantificación de resultados. La aplicación del enfoque constructivista traspasa las tramas del discurso gerencial del evaluador y del discurso legitimador de la DGII. Es la vía por la que acceden al texto otras fuerzas, otros discursos. Induce aperturas por las que se instalan en el texto otros sentidos.

Será en la recepción del informe donde las tensiones reproducidas en el texto se manifiesten materialmente. En la lectura, como momento de realización del texto $^{35}$, observamos las energías que este va a concitar y desplegar.

\subsubsection{La recepción}

En la recepción acontece el encuentro entre el texto y sus destinatarios, entre el autor y los lectores, entre el discurso evaluativo y la instancia de gobierno que ha promovido la evaluación. Se reproduce la situación de partida, los encuentros que tuvieron lugar durante el proceso de formación de la demanda. Hay un reencuentro en el que se hacen materialmente sensibles las tensiones del campo y los desacuerdos, algunos latentes, de esos primeros encuentros. En el proceso de la evaluación, decíamos, hay un encadenamiento de desfases progresivamente amplificados. En la recepción se van a manifestar como despliegue material de fuerzas.

La recepción se constituye como un proceso de lectura y de diálogo en torno al texto del informe. Ese diálogo desemboca en una lucha por la apropiación del texto y por su verdad. Lo escrito dio lugar a un intercambio de energías entre los destinatarios del informe y su autor, una lucha que seguirá cauces no dialógicos. Lo dicho por los otros, las valoraciones de los agentes territoriales, fueron la fuente de un conflicto que se prolongó en intentos de imponer otro sentido al informe, de reescribirlo: presión del dispositivo de gobierno sobre el texto, "tentativas de captura" del evaluador. El poder gubernamental que había instado el informe trató de operar sobre él: había que suprimir lo que no podía aparecer por escrito y sobre-representar lo que sí debía aparecer, lo favorable a la gerencia.

La apertura constructivista produjo un efecto de liberación discursiva, relativo y momentáneo, que había que controlar. Fue una apertura limitada, pero, aun así, lo dicho por los agentes era fuente de peligro. Tal como consta en el cuaderno de notas del evaluador, las valoraciones de los agentes daban "miedo". De ahí los intentos de apropiación del texto y de censura que acontecieron en la recepción del informe: negar la verdad de los agentes, delimitar lo que no se podía decir y lo que se debía decir ${ }^{36}$.

${ }^{35}$ En el sentido que Paul Ricoeur (1999 [1986]: 81) da a la lectura: "acto concreto en el que se cumple el destino del texto".

36 En la reunión de presentación del informe (de 19 de mayo de 2008), de carácter interno y restringido, los intentos de imponer un nuevo sentido al texto adoptaron cuatro formas: 1 . La degradación del estatus epistemológico de lo dicho por los agentes: tal como manifestó una de las responsables de la DGII, eran "valoraciones, no hechos objetivos", no tenían estatuto de cosas ciertas (cuestionamiento del régimen de veridicción del texto). 2. En relación con lo anterior, la 
Los agentes habían cuestionado el modelo de gestión del Fondo de Apoyo y el marco normativo que regulaba la inmigración, la Ley de extranjería. En ese marco legal, las acciones financiadas por el FAIRE corrían el riesgo de ser ineficaces como instrumentos de integración social. Lo que dijeron los actores entrevistados, y lo que el texto de la evaluación transcribió, es que la Ley producía la exclusión, que la Ley era contradictoria con respecto a los fines del FAIRE. El límite de la escritura, la frontera de lo políticamente admisible, estaba demarcada por la verdad de la Ley. Al igual que sucedió cuando se constituyó la política de integración de inmigrantes, la Ley de extranjería, previa a dicha política, era intangible: la veridicción jurídica quedaba fuera del alcance de la veridicción técnica.

La apertura plural de la evaluación dio lugar a una crítica que contravino radicalmente las expectativas de la DGII. Ese fue el efecto final de la apertura. Lo que provocó el miedo y la contra-respuesta de la gerencia, sus intentos de apropiación del texto. La recepción estuvo condicionada por una expectativa previa, por un deseo de legitimación anterior al texto. La DGII pretendió actualizar el discurso de la demanda: había una pre-determinación de lo que debía ser escrito, un sentido que ya se había decidido. En la lectura, se rechazó lo que no respondía a esa expectativa previa, lo que no servía para realizar ese deseo anterior.

Estas luchas en torno al texto se resolvieron de un modo incierto, sin un vencedor claro. Finalmente, el informe no se modificará. Sin embargo, se elaborará una segunda versión, abreviada, un Resumen ejecutivo (GPI, 2008b) pensado para su difusión institucional, que incorporará la mayor parte de los cambios solicitados por la DGII: eliminación total de las transcripciones del decir de los agentes y de las referencias críticas al marco legal de la inmigración. Tampoco este resumen será difundido.

\subsection{ENSEÑANZAS DEL CASO}

Frente a la concepción convencional (y tecnocrática) del informe de investigación/evaluación como mensaje que la naturaleza, capturada por el investigador, envía a través de este, y frente a su supuesto epistemológico, el emplazamiento del investigador en un punto arquimédico, externo al objeto investigado, el caso nos muestra:

\footnotetext{
puesta en cuestión de la verdad de los agentes, su falta de correspondencia con la realidad: eran "respuestas simples a problemas complejos"; el texto debía "ajustarse más a la realidad" (cuestionamiento de la verdad del texto). 3. La reclamación de un sentido institucionalmente favorable: "reflejar más lo positivo" (lucha por el sentido general del texto). 4. En el límite, la censura: la transcripción del discurso valorativo de los agentes debía eliminarse del informe ("esto no lo puedo poner en un informe del Ministerio"); la pertinencia de la intervención no debía ser objeto de evaluación (lucha por el derecho de lo escrito a aparecer en el texto). Las entrevistas con el evaluador y sus notas, tomadas durante la reunión, permiten reconstruir estas apreciaciones y requerimientos de la DGII.
} 
- Que la investigación es, ante todo, un proceso de escritura.

- Que el investigador es el punto de aplicación de una serie de fuerzas previas (discursivas, sociales, políticas) que actúan sobre su escritura, la fragmentan y dispersan sus sentidos.

- Que en la recepción del informe se revelan, materialmente, los desacoplamientos entre discursos-prácticas de investigación y discursosprácticas gubernamentales. La sobredeterminación política y social del texto no implica que este sea, necesariamente, una mera reproducción ideológica de un dispositivo de gobierno.

Desarrollamos, brevemente, estos argumentos:

1. Un informe es el resultado de un proceso en el que se encadenan varias operaciones: de formación de la demanda, de diseño de investigación, de generación de información y, finalmente, de escritura. Esas operaciones no siguen una continuidad lineal. Están mediadas por "el trabajo del texto", configuradas por una escritura que las teje y las desteje. Entre el diseño, la producción de información y el resultado/informe no hay un encadenamiento simple y mecánico.

En el caso, el diseño de la evaluación, lo teóricamente previo a su realización, se transforma en el trabajo de la escritura. Es en el texto, en la superficie de inscripción del informe, donde toman forma definitiva las decisiones relativas a la formulación de objetivos, conceptos y criterios de valor de la evaluación. La formación del régimen conceptual y valorativo de la investigación y la escritura del informe convergen en un mismo proceso; como si, a la luz de los materiales recabados, de sus posibilidades analíticas, de las fuerzas actuantes en el proceso de la evaluación, la escritura tejiera, recursivamente, su orden categorial y axiológico. La investigación se realiza, ante todo, en el texto mismo.

2. Esa escritura es, materialmente, producto de un autor, pero su estatus se transforma al escribir. En el caso, el autor/evaluador no habla desde un único lugar, se desplaza operando cambios en su posición respecto a lo dicho en el informe: a veces, ratifica el discurso del cliente; otras, le juzga con la autoridad de un juez experto; otras, simplemente, calla ante el decir crítico de los agentes... Esos cambios enunciativos correlacionan con sus propias posiciones en un campo de relaciones y fuerzas exterior al texto:

- El evaluador es un sujeto-sujetado en una relación comercial asimétrica. Debe plegarse a los intereses de legitimación del cliente y escribir un relato que ratifique su discurso, la representación que la gerencia ha hecho de sí misma.

- Sin embargo, el evaluador es, ante todo, sujeto-experto, sujeto que sabe y que debe juzgar la labor del cliente desde los criterios tecno-económicos de la racionalidad gerencial. Su discurso, en tanto que discurso experto, se autonomiza con respecto a los intereses de legitimación del cliente. Está obligado por esa racionalidad técnica a la que sirve y que 
habla a través de él.

- Por último, el evaluador está comprometido con la metodología (dialógica, pluralista) que ha elegido, y por unos actores, los agentes territoriales, a los que ha convocado. Debe llevar su discurso al texto, que será, finalmente, una frágil solución de compromiso entre esas fuerzas diversas que actúan sobre él.

Por eso, el informe, más que un producto acabado, clausurado, es un "proceso de significaciones en operación" (Barthes, 1990: 323). La unidad del texto es precaria, solo una apariencia primera. Bajo la forma, administrativamente codificada, de un informe, el texto se ramifica en una multiplicidad de voces, de sentidos, a veces contradictorios, enfrentados... Sentidos abiertos conjugados por un autor que, de forma correlativa, no habla desde una posición unificada.

La escritura del informe evoca, entonces, "el combate de los hombres y de los signos": el autor se esfuerza en reducir "lo plural del texto, la apertura de su significancia" (Ibíd.: 324). Trata de asegurar su coherencia, aunque no puede lograrlo del todo. Lucha del autor con el texto, tanto más agónica cuanto se da en un tipo de discurso cuyo horizonte ideal es la univocidad, en un género empeñado en negar la indecidibilidad del sentido: el texto tecnocrático, orientado a demostrar la irrevocabilidad del one best way de la acción de gobierno.

3. La recepción de un informe delimita el espacio de un encuentro entre discursos y prácticas. El texto circula materialmente en el interior del dispositivo gubernamental que ha instado su producción. Pero no es una mera reproducción discursiva de ese dispositivo: entre el discurso y la práctica evaluativas y entre los discursos y las prácticas gubernamentales no hay una homología estricta, un acoplamiento mecánico. Lo que encontramos es, como diría Deleuze (1987: 9596), su "anisomorfía", un juego de "insinuaciones recíprocas", de "presiones y capturas mutuas" entre formas que son heterogéneas, irreductibles entre sí.

En la recepción del informe de evaluación del FAIRE se pone de manifiesto, energéticamente, un desencuentro radical entre el discurso de los agentes territoriales, cuyo decir ha sido llevado al texto por el evaluador, y el discurso de la demanda (las expectativas de la DGII, finalmente frustradas). Ese desacoplamiento entre el discurso evaluativo y el dispositivo gubernamental motiva las presiones de este, sus intentos de imponer otro sentido al texto, de reescribirlo, de clausurarlo...

Lo que acontece en el espacio concreto, históricamente puntual, de la recepción del informe de evaluación del FAIRE está sobredeterminado por otros desacoplamientos, de mayor profundidad y alcance:

- En el interior del dispositivo de gobierno operan diferentes racionalidades, discursos y prácticas, cada una de ellas con su propia lógica, su propio centro: hay una razón jurídico-burocrática heredada, una razón político-partidista y una razón gerencial, en la que se inscribe el discurso de la evaluación. Y este, asumido formalmente por el dispositivo guber- 
namental, es solo un discurso más que trata de abrirse camino desplazando a los otros discursos, a las otras lógicas (en realidad mucho más arraigadas, más poderosas).

- Pero también la evaluación, como formación discursiva, alberga su propia dispersión interna. En el nivel de la teoría, el discurso evaluativo está atravesado por una serie de difracciones, históricamente generadas. Estas difracciones introducen tensión en las prácticas de la evaluación y en sus textos. Esto es lo que sucede en el caso.

El texto del informe de evaluación del Fondo de Apoyo se escribió desde una encrucijada. Al pretender articular perspectivas teórico-metodológicas divergentes y, sobre todo, al optar por un enfoque pluralista y dialógico, llevó al límite el desacoplamiento entre el discurso evaluativo y el dispositivo de gobierno. Y sucedió algo más: se situó allí donde la evaluación cristaliza como práctica y encuentra, a la vez, sus tensiones fundamentales (también sus aporías).

En la formación del discurso evaluativo se plantean muy pronto los problemas de la apertura y la participación plural. La pluralidad sociopolítica empuja y modela el discurso de la evaluación, lo abre en la difracción teórico-metodológica fundamental que enfrenta enfoques dialógicos con enfoques gerenciales. Pero esos enfoques que se abren y fragmentan el campo evaluativo son algo más que opciones teórico-metodológicas. Son, en último término, representaciones de la sociedad y del poder. Esa encrucijada teórica es, finalmente, una metáfora de nuestros propios dilemas sociopolíticos: gobierno de los expertos/democracia deliberativa, representación cerrada/gobierno abierto, gubernamentalidad técnica/orden jurídico-burocrático/lógica de partido...

La polifonía discursiva del informe, los sentidos abiertos por su escritura, las tensiones que no puede resolver y la clausura final de sus usos nacen en esta encrucijada teórica, social y política, desde la que se ha escrito el texto y que se actualiza en él.

\section{REFERENCIAS BIBLIOGRÁFICAS}

AUGÈ, M. (2004): ¿Por qué vivimos? Por una antropología de los fines. Barcelona, Gedisa.

BARTHES, R. (1990): La aventura semiológica. Barcelona, Paidós.

CAMPBELL, D. T. (1969): "Reforms as experiments", American Psychologist, 24, pp. 400-429.

-(1975): “Assesing the impact of planned social change", en G.M. Lyons (ed.): Social

Research and Public Policies. Darmouth College, NH, Publics Affairs Center, pp. 3-45.

-(1982): "Experiments as arguments", en E. R. House, S. Mathison, J. A. Pearsol y H. Preskill (eds.), Evaluation Studies Review Anual, Vol. 7, pp. 117-128.

DEL ÁGUILA, R. (coord.) (2005): La inmigración. Un desafio para España. Madrid, Editorial Pablo Iglesias.

DELEUZE, G. (1987): Foucault. Barcelona, Paidós. 
DU GAY, P. (1994): "Making up managers: bureaucracy, enterprise and the liberal art of separations", British Journal of Sociology 45 (4), pp. 655-674.

-(2000): In Praise of Bureaucracy. Weber, Organization, Ethics. Londres, Sage.

FOUCAULT, M. (1969) : L'archéologie du savoir. París, Gallimard.

-(1981): “Omnes et singulatim. Toward a Criticism of 'Political Reason"”, en The Tanner Lectures on Human Values, II. Salt Lake City, University of Utah Press, pp. 223254.

-(2004): Naissance de la biopolitique. Cours au Collège de France. 1978-1979. París: Seuil/Gallimard. (Trad. cast.: Nacimiento de la biopolítica. Curso del Collège de France (1978-1979). Madrid, Akal, 2009.)

GARDE ROCA, J. A. (2006). "La evaluación de políticas públicas y su institucionalización en España", Papeles de evaluación, 3, pp. 1-33.

GPI CONSULTORES (2007): Diseño del modelo de evaluación del Fondo de Apoyo para la Acogida y la Integración de los Inmigrantes-2006. Madrid, Dirección General de Integración de los Inmigrantes, Ministerio de Trabajo y Asuntos Sociales.

-(2008a) Evaluación del Fondo de Apoyo para la Acogida y la Integración de los inmigrantes-2006. Madrid, Dirección General de Integración de los Inmigrantes, Ministerio de Trabajo y Asuntos Sociales.

-(2008b): Resumen ejecutivo de la evaluación del Fondo de Apoyo para la Acogida y la Integración de los inmigrantes-2006. Madrid: Dirección General de Integración de los Inmigrantes, Ministerio de Trabajo y Asuntos Sociales.

GUBA, E. G. y LINCOLN, B. S. (1986): "The Countenances of Fourth Generation Evaluation: Description, Judgement, and Negotiation", Evaluation Studies Review Annual, Vol. 11, pp. 70-80.

-(1989): Fourth Generation Evaluation. Newbury Park, Sage.

HOUSE, E. R. (1979): “The objectivity, fairness, and justice of federal evaluation policy as reflected in the Follow Through evaluation", Educational Evaluation and Policy Analysis, Vol. 1, pp. 28-42.

-(1980): Evaluating with Validity. Beverly Hills, CA: Sage. (Trad. cast.: Evaluación, ética y poder. Madrid, Morata, 2000.)

HOUSE, E. R. y HOWE, K. R. (1999): Values in Evaluation and Social Research. Beverly Hills, CA: Sage. (Trad.cast.: Valores en evaluación e investigación social. Madrid, Morata, 2001.)

IBÁÑNEZ, J. (1986): "El diseño en la perspectiva estructural", en F. Alvira, M. G. Ferrando y J. Ibáñez (comps.): El análisis de la realidad social: métodos y técnicas de investigación. Madrid, Alianza, pp. 31-66.

JENSEN, M. C. y MECKLING, W. H. (1976): "Theory of the firm: managerial behavior, agency costs and capital structure", Journal of Financial Economics, 3(4), pp. 305-360.

QUADE, E. S. (1982): Analysis for public decisions. New York, Elsevier Science Publising Co.

REYNOLDS, J. y GASPARI, K. C. (1985): Cost-effectiveness analysis. Chevy Chase, PRICOR, Primary Health Care Operarions Research, Center for Human Services.

RICOEUR, P. (1969): Le conflit des interprétations. París, Seuil.

-(1971): "The model of the text. Meaningful action considered as a text", Social Research, 38, pp. 529-562. 
-(1986): “Qu'est-ce qu'un texte?”, en P. Ricoeur: Du texte á l'action. Essais d'herméneutique II. París, Seuil, pp. 137-160. (Trad. cast.: “¿Qué es un texto?”, en P. Ricoeur: Historia y narratividad. Barcelona, Paidós, ICE/UAB, 1999, pp. 59-81.)

RIVLIN, A. M. (1971): Systematic Thinking for Social Action. Washington, DC, Brookings Institution.

Rivlin, A. M. y TIMPANE, M. M. (eds.) (1975): Planned Variations in Education. Washington, DC, Brookings Institution.

ROSSI, P. H., FREEMAN, H. E. y WRIGHT, S. R. (1979): Evaluation. A Systematic Approach. Beverly Hills, Sage.

SCRIVEN, M. (1972): "Objectivity and subjectivity in educational research", en L. G. Thomas (ed.): Philosophical Redirection of Educational Research. Chicago, National Society for the Study of Education, pp. 94-142.

-(1973): "Goal free evaluation", en E. R. House (ed.): School Evaluation: The Politics and Process. Berkeley, CA, McCutchan, pp. 319-328.

-(1976): "Evaluation biass and its control", en G. V. Glass (ed.): Evaluation Studies Review Annual, Vol. 1, pp. 119-139.

SHAW, I.F. (1999): Qualitative Evaluation. Londres, Sage. (Trad. cast.: La evaluación cualitativa. Introducción a los métodos cualitativos. Barcelona, Paidós, 2003.)

SMITH, E. y TYLER, R. W. (1942): Appraising and Recording Student Progress. New York, Harper \& Row.

STAKE, R. E. (1976). Evaluating Educational Programmes: The Need and the Response. Washington, DC, OECD Publications Center.

-(1978): "The case study method in social inquiry", Educational Researcher, 7, pp. 5-8.

STAKE, R. E. y EASLEY, J. (1978): Case Studies in Science Education. Urbana, Center for Instructional Research and Curriculum Evaluation, University of Illinois.

TYLER, R. W. (1950): Basic Principles of Curriculum and Instruction. Chicago, University of Chicago Press.

VAN DIJK, T. (2000): "El discurso como interacción en la sociedad", en T. van Dijk (comp.) El discurso como interacción social. Estudios sobre el discurso II. Una introducción multidisciplinaria. Barcelona, Gedisa, pp. 19-66. 
